\title{
Transport Phenomena and Light Element Abundances in the Sun and Solar Type Stars
}

\author{
Sylvie Vauclair
}

Laboratoire d'Astrophysique, 14 av. Ed. Belin, 31400-Toulouse, France

\begin{abstract}
.
The observations of light elements in the Sun and Solar type stars give special clues for understanding the hydrodynamical processes at work in stellar interiors. In the Sun ${ }^{7} \mathrm{Li}$ is depleted by 140 while ${ }^{3} \mathrm{He}$ has not increased by more than $\cong 10 \%$ in 3 Gyrs. Meanwhile the inversion of helioseismic modes lead to a precision on the sound velocity of about .1\%. The mixing processes below the solar convection zone are constrained by these observations. Lithium is depleted in most Pop I solar type stars. In halo stars however, the lithium abundance seems constant in the "spite plateau" with no observed dispersion, which is difficult to reconcile with the theory of diffusion processes. In the present paper, the various relevant observations will be discussed. It will be shown that the $\mu$-gradients induced by element settling may help solving the "lithium paradox".
\end{abstract}

\section{Introduction}

Element diffusion and mixing processes in stellar interiors are now widely constrained, first by detailed observations of abundances, second by helio and asteroseismology. In most cases however, pure microscopic diffusion in stars would lead to abundance variations much larger than those observed : mild macroscopic motions in stellar radiative zones are definitely needed to account for the observations. This gives strong constraints on the kind of mixing processes allowed. Other constraints come from the consequences of the nuclear reactions occuring in stellar interiors : in some cases stellar mixing from the atmosphere down to the regions of nuclear processing is needed to explain the observed element abundances. This is the case, for example, to account for the depletion of lithium in the Sun and solar type stars.

Lithium observations in main-sequence population I field stars and galactic clusters show a large abundance dispersion which has been extensively studied in the literature (see reviews by Deliyannis 2000, Charbonnel 2000, Michaud 2000 and Pinsonneault 2000). The lithium abundance decreases for decreasing effective temperature below $5500 \mathrm{~K}$ and the depletion increases with increasing age. This is generally attributed to the deepening of the convective zone, associated with some mild mixing process connecting the bottom of the convective zone with the nuclear destruction region.

Lithium is also depleted in F-type stars (the so-called "Boesgaard dip"). Several possible reasons have been invoked to explain this feature, most related 
to mixing and nuclear destruction. Element segregation has been proved negligible here as it would lead to unobserved variations of metal abundances (Turcotte et al 1998) and beryllium (Boesgaard 2000).

On the other hand, observations of lithium in main-sequence population II field stars show remarkably constant abundances, with a very small dispersion (e.g. Bonifacio and Molaro 1997) Why is lithium destroyed in Pop I stars while it does not seem destroyed in Pop II stars?

For the same effective temperatures, the convective zone is smaller in Pop II stars than in Pop I stars because of their smaller metallicity. Meanwhile they have a smaller rotation velocity on the average. This could explain why the lithium destruction induced by nuclear reactions is smaller in these stars than in Pop I stars. However the element segregation is more important for smaller densities and smaller rotation, so that this process should lead to a visible lithium depletion, which is not observed (Vauclair and Charbonnel 1995 and 1998). This represents the so-called "lithium paradox". Here we suggest that the influence of $\mu$-gradients on the rotation-induced mixing may help solving this paradox.

\section{Competition between rotation induced mixing and element diffu- sion}

In rotating stars, the equipotentials of "effective gravity" (including the centrifugal acceleration) have ellipsoidal shapes while the energy transport still occurs in a spherically symetrical way. The resulting thermal imbalance must be compensated by macroscopic motions: the so-called "meridional circulation" (Von Zeipel 1924). The stellar regions outside the convective zones cannot be in complete radiative equilibrium. They are subject to entropy variations given by :

$$
\begin{aligned}
\rho T\left(\frac{\partial S}{\partial t}+\mathbf{u} \cdot \nabla S\right) & =-\nabla \cdot \mathbf{F}+\rho \varepsilon_{n} \\
& =\rho \varepsilon_{\Omega}(\neq 0)
\end{aligned}
$$

where $\mathbf{F}$ represents the heat flux, $\varepsilon_{n}$ the nuclear energy production and $\varepsilon_{\Omega}$ an energy generation rate which results from sources and sinks of energy along the equipotentials.

The vertical component of the meridional velocity $u_{r}$ is computed as a function of $\varepsilon_{\Omega}$ in the stationary regime (from eq. 1):

$$
u_{r}=\left(\frac{P}{C_{p} \rho T}\right) \frac{\varepsilon_{\Omega}}{g}
$$

which, for a perfect gas, reduces to:

$$
u_{r}=\frac{\varepsilon_{\Omega}}{g} \frac{\nabla_{\mathrm{ad}}}{\nabla_{\mathrm{ad}}-\nabla+\nabla_{\mu}}
$$

where $g$ represents the local gravity, $\nabla_{\text {ad }}$ and $\nabla$ the usual adiabatic and real ratios $\left(\frac{d \ln T}{d \ln P}\right)$ and $\nabla_{\mu}$ the mean molecular weight contribution $\left(\frac{d \ln \mu}{d \ln P}\right)$. 
The expression of $\varepsilon_{\Omega}$ is computed by expanding the right-hand-side of eq. (1) on a level surface and writing that its mean value vanishes.

Mestel (1953, 1957 and 1965) pointed out that, in the presence of vertical $\mu$-gradients, $\varepsilon_{\Omega}$ contains two kinds of terms : those related to the resulting horizontal variations of $\mu$ : the so-called " $\mu$-induced currents" $E_{\mu}$ and those independent of $\mu$, the so-called " $\Omega$-induced currents" $E_{\Omega}$. The expression of $\varepsilon_{\Omega}$ obtained in this case has been derived in detail by Maeder and Zahn (1998), who took into account several effects which were not included in the previous computations: more general equations of state instead of perfect gas law, presence of a thermal flux induced by horizontal turbulence, non-stationary cases.

Vauclair (1999) discussed more simple expressions, valid only for negligible differential rotation. In this case $\mu$-currents are opposite to $\Omega$-currents in most of the star and $\varepsilon_{\Omega}$ may be written :

$$
\varepsilon_{\Omega}=\left(\frac{L}{M}\right)\left(E_{\Omega}+E_{\mu}\right) P_{2}(\cos \theta)
$$

with:

$$
\begin{aligned}
& E_{\Omega}=\frac{8}{3}\left(\frac{\Omega^{2} r^{3}}{G M}\right)\left(1-\frac{\Omega^{2}}{2 \pi G \bar{\rho}}\right) \\
& E_{\mu}=\frac{\rho_{m}}{\bar{\rho}}\left\{\frac{r}{3} \frac{d}{d r}\left[\left(H_{T} \frac{d \Lambda}{d r}\right)-\left(\chi_{\mu}+\chi_{T}+1\right) \Lambda\right]-\frac{2 H_{T} \Lambda}{r}\right\}
\end{aligned}
$$

Here $\bar{\rho}$ represents the density average on the level surface $(\simeq \rho)$ while $\rho_{m}$ is the mean density inside the sphere of radius $r ; H_{T}$ is the temperature scale height; $\Lambda$ represents the horizontal $\mu$ fluctuations $\frac{\tilde{\mu}}{\bar{\mu}} ; \chi_{\mu}$ and $\chi_{T}$ represent the derivatives:

$$
\chi_{\mu}=\left(\frac{\partial \ln \chi}{\partial \ln \mu}\right)_{P, T} \quad ; \quad \chi_{T}=\left(\frac{\partial \ln \chi}{\partial \ln T}\right)_{P, \mu}
$$

Vertical $\mu$-gradients may occur in stars due to two different processes : first the nuclear reactions which occur in the stellar cores, second the helium settling which occurs in the outer layers. The importance of the first process in reducing or even suppressing the meridional motions has been demonstrated several times in the literature (e.g. Huppert and Spiegel 1977). The second process on the other hand has not been extensively studied. We claim here that it may play a crucial role for understanding the lithium problem in Pop I and Pop II stars.

\section{Application to Pop II stars}

Computations of $\mu$-currents induced by the helium settling in halo stars have been performed by Vauclair 1999 and Théado and Vauclair 2000 a and b. We found that, for slow rotation, $\mu$-currents cancel $\Omega$-currents for very small concentration gradients, corresponding to $\mu$-gradients of order $10^{-15} \mathrm{~cm}^{-1}$.

Let us summarize the situation of a slowly rotating star in which element settling leads to an increase of the $\mu$-gradient below the outer convection zone. At the beginning, the star is homogeneous and meridional circulation can occur, 
leading to upward flows in the polar regions and downward flows in the equatorial parts (except in the very outer layers where the Gratton-Öpik term becomes important, which we do not discuss here). The $\mu$-currents, opposite to the classical $\Omega$-currents, are first negligible. The $\mu$-gradients increasing with time because of helium settling, the order of magnitude of the $\mu$-currents also increases until it reaches the value for which the circulation vanishes.

This does not occur all at once: as the $\mu$-gradient decreases with depth below the convective zone, we expect that the meridional circulation freezes out step by step (see figure 1 of Théado and Vauclair 2000a). An equilibrium situation may be reached, in which the temperature and mean molecular weight gradients along the level surfaces are such that $\Omega$-currents and $\mu$-currents cancel each other.

Once it is reached, this equilibrium situation is quite robust. Suppose that some mechanism leads to a decrease of the $\mu$-gradient: then $\left|E_{\mu}\right|$ becomes smaller than $\left|E_{\Omega}\right|$ and the circulation tends to be restablished in the $\left|E_{\Omega}\right|$ direction, thereby restoring the original $\mu$ gradient. Suppose now that the $\mu$-gradient is increased. Then $\left|E_{\mu}\right|$ becomes larger than $\left|E_{\Omega}\right|$ and the circulation begins in the $E_{\mu}$ direction. Here again the original gradient is restored.

When the meridional circulation is frozen below the convective zone, helium settling could proceed further; however, due to the increase of the diffusion time scale with depth, this would modify the $\mu$-gradient. We may thus expect that $\mu$ currents would take place and restore the original equilibrium gradient, thereby strongly reducing the microscopic diffusion (Théado and Vauclair 2000b). This self-regulating process could be the reason for the low dispersion of the lithium abundance in the lithium plateau of halo stars.

\section{Discussion : Pop I versus Pop II stars}

There are many observations in stars which give evidences of mixing processes occuring below the outer convective zones as, for example, the lithium depletion observed in the Sun and in galactic clusters. The process we have described above should not apply in all these stars. The reason could be related to the rapid rotation of young stars on the ZAMS and to their subsequent rotational braking.

The abundance determinations in the solar photosphere show that lithium has been depleted by a factor of about 140 compared to the protosolar value while beryllium has not been depleted by more than a factor 2, and maybe much less, as discussed by Balachandran and Bell (1997). These values represent strong constraints on the mixing processes in the solar interior.

Observations of the ${ }^{3} \mathrm{He} /{ }^{4} \mathrm{He}$ ratio in the solar wind and in the lunar rocks (Geiss 1993, Geiss and Gloecker 1998) show that this ratio may not have increased by more than $\cong 10 \%$ since $3 \mathrm{Gyr}$ in the Sun. While the occurence of some mild mixing below the solar convective zone is needed to explain the lithium depletion, the ${ }^{3} \mathrm{He} /{ }^{4} \mathrm{He}$ observations put a strict constraint on its efficiency. The only way to obtain such a result is to postulate a mild mixing, which would be efficient down to the lithium nuclear burning region but not too far below, to preserve the original ${ }^{3} \mathrm{He}$ abundance. The efficiency of this mixing 
should also decrease with time, as the ${ }^{3} \mathrm{He}$ peak itself builts up during the solar life.

It is interesting to compute the minimum enhancement of the ${ }^{3} \mathrm{He} /{ }^{4} \mathrm{He}$ ratio implied by the lithium observed depletion. Vauclair and Richard 1998 showed that it is possible to deplete lithium by a factor larger than 100 as observed and not increase ${ }^{3} \mathrm{He} /{ }^{4} \mathrm{He}$ by more than 5 percent since the solar origin. In this case beryllium is only depleted by about 10 percent.

Such a confined mixing zone is also needed from helioseismology : although the introduction of pure element settling in the solar models considerably improves the consistency with the seismic Sun, some discrepancies do remain, particularly below the convective zone where a "spike" appears in the sound velocity (Richard et al 1996, Turck-Chièze et al. 1998). It has been shown that this behavior may be due to the helium gradient which would be too strong in case of pure settling. Mild macroscopic motions below the convective zone slightly decrease this gradient and helps reducing the discrepancy (Richard et al 1996, Corbard et al 1998, Brun et al 1998). The helium profiles directly obtained from helioseismology (Basu 1998, Antia and Chitre 1998) show indeed a helium gradient smoother than the gradient obtained with pure settling.

The constraints implied by both the helioseismic inversions and abundance determinations in the Sun converge towards the existence of a small mild mixing region below the convective zone, which would extend down to a depth of the order of one scale height. The implied mixing region must be very mild, with diffusion coefficients of $10^{3}-10^{4}$ only. It must also be completely deconnected from the solar core. No mixing can indeed be allowed down to the nuclear energy production region as it would lead to a sound velocity incompatible with helioseismology. In particular the mixing processes invoked by Morel and Schatzman 1996 to decrease the neutrino fluxes are excluded by helioseismology (Richard and Vauclair 1997).

Mixing processes localized at the boundary between convective and radiative regions include overshooting and regions of large differential rotation like the "tachocline" below the solar convective zone. Up to now, overshooting was generally treated in the models simply as a continuation of the convective zone on a fraction of a pressure scale height. Recent parametrisations use a diffusion coefficient which decreases exponentially with decreasing radius (Freytag et al 1996). The tachocline, which represents in the present Sun the small boundary between the region of large differential rotation (in the convective zone) and the region of solid rotation (in the radiative zone below) is also treated as a mixed layer with an exponentially decreasing diffusion coefficient (Brun et al 1998, Richard 1999). Results are encouraging, although more sophisticated numerical simulation including $2-\mathrm{D}$ abundance variations would be needed to go further.

In any case, the self-regulating process that we have discussed for halo stars in section 3 would not apply below the convective zone in the Sun and solar type stars because of the differential rotation which takes place there. Such a differential rotation would not be expected in halo stars if we suppose that they always rotated slowly and thus did not suffer large transport of angular momentum. The different behavior for the lithium abundance in Pop I and Pop II stars could thus be directly related to their rotation history. 


\section{References}

Antia, H.M., Chitre, S.M., 1998, $A \& A$ 339, 239

Balachandran, S.C., Bell, R.A., 1997, American Astronomical Society Meeting 191, 7408

Basu, S., 1998, M.N.R.A.S. 298, 719

Boesgaard, A.M., 2000, to be published in The 11th Cambridge Workshop on cool stars, stellar systems and the sun, Challenges for the New Millenium

Bonifacio P., Molaro P., 1997, MNRAS, 285, 847

Brun, A.,S., Turck-Chieze, S., Morel, P., 1998, ApJ 506, 113

Charbonnel, C., 2000, this meeting

Corbard,T., Berthomieu, G., Provost, P., Morel, P., 1998, A\&A 330, 1149

Deliyannis, C., 2000, this meeting

Freytag,B., Ludwig,H., Steffen M., 1996, $A \& A$ 313, 497

Geiss, J.: 1993, Origin and Evolution of the Elements, ed. Prantzos, VangioniFlam \& Cassé (Cambridge Univ. Press), 90

Geiss, J., Gloecker, G., 1998, Space Science Reviews 84, 239

Huppert, H.E., Spiegel, E.A., 1977, $A p J$ 213, 157

Maeder A., Zahn J.-P., 1998, A $\circlearrowleft A$ 334, 1000

Mestel L., 1953, M.N.R.A.S. 113, 716

Mestel L., 1957, ApJ 126, 550

Mestel L., 1965, Stellar Structure, in Stars and StellarSystems, vol 8, ed. G.P. Kuiper, B.M. Middlehurst, Univ. Chicago Press, 465

Michaud, G., 2000, this meeting

Pinsonneault, M., 2000, this meeting

Richard, O., 1999, PhD thesis, University of Toulouse

Richard, O., Vauclair, S., Charbonnel, C., Dziembowski, W.A., 1996, $A \& A$ 312, 1000

Richard, O., Vauclair, S., 1997, $A \& A$ 322, 671

Théado, S., Vauclair, S., 2000 a, this meeting

Theado, S., Vauclair, S., 2000 b, preprint

Turck-Chieze, S., Basu, S., Berthomieu, G., Bonnano, A., Brun, A.S., ChristensenDalsgaard, J., Gabriel, M., Morel, P., Provost, J., Turcotte, S., The Golf Team, 1998, in Structure and Dynamics of the Interior of the Sun and Sun-like Stars ESA Publications Division, SP-418, 555

Turcotte, S., Richer, J., Michaud, G. Iglesias, C.A., Rogers, F.J., 1998, ApJ $\mathbf{5 0 4}, 539$

Vauclair, S., 1999, $A \& A$ 351, 973

Vauclair, S., Charbonnel, C., 1995, $A \& A$ 295, 715

Vauclair, S., Charbonnel, C., 1998, ApJ 502, 372

Vauclair, S., Richard, O.: 1998, in Structure and Dynamics of the Interior of the Sun and Sun-like Stars ESA Publications Division, SP-418, 427

Von Zeipel H., 1924, M.N.R.A.S. 84, 665 